\title{
Description of call handling in emergency medical dispatch centres in Scandinavia: recognition of out-of-hospital cardiac arrests and dispatcher-assisted CPR
}

\author{
Camilla Hardeland ${ }^{1,2^{*}}$ (D), Andreas Claesson ${ }^{3}$, Marieke T. Blom ${ }^{4}$, Stig Nikolaj Fasmer Blomberg ${ }^{5}$, Fredrik Folke ${ }^{5}$, \\ Jacob Hollenberg ${ }^{3}$, Jo Kramer-Johansen², Freddy Lippert ${ }^{5}$, Anette Nord ${ }^{3}$, Anne Mette Nygaard ${ }^{1}$, \\ Theresa Mariero Olasveengen ${ }^{6}$, Mattias Ringh ${ }^{3}$, Leif Svensson ${ }^{7}$ and Thea Palsgaard Møller ${ }^{5}$
}

\begin{abstract}
Background: The European resuscitation council have highlighted emergency medical dispatch centres as an important key player for early recognition of Out-of-Hospital Cardiac Arrest (OHCA) and in providing dispatcher assisted cardiopulmonary resuscitation (CPR) before arrival of emergency medical services. Early recognition is associated with increased bystander CPR and improved survival rates. The aim of this study is to describe OHCA call handling in emergency medical dispatch centres in Copenhagen (Denmark), Stockholm (Sweden) and Oslo (Norway) with focus on sensitivity of recognition of OHCA, provision of dispatcher-assisted CPR and time intervals when CPR is initiated during the emergency call (NO-CPR prior), and to describe OHCA call handling when CPR is initiated prior to the emergency call (CPR prior).

Methods: Baseline data of consecutive OHCA eligible for inclusion starting January 1st 2016 were collected from respective cardiac arrest registries. A template based on the Cardiac Arrest Registry to Enhance Survival definition catalogue was used to extract data from respective cardiac arrest registries and from corresponding audio files from emergency medical dispatch centres. Cases were divided in two groups: NO-CPR prior and CPR prior and data collection continued until 200 cases were collected in the NO-CPR prior-group.

Results: NO-CPR prior OHCA was recognised in $71 \%$ of the calls in Copenhagen, $83 \%$ in Stockholm, and 96\% in Oslo. Abnormal breathing was addressed in 34,7 and 98\% of cases and CPR instructions were started in 50,60, and 80\%, respectively. Median time (mm:ss) to first chest compression was 02:35 (Copenhagen), 03:50 (Stockholm) and 02:58 (Oslo). Assessment of CPR quality was performed in 80, 74, and $74 \%$ of the cases. CPR prior comprised 71 cases in Copenhagen, 9 in Stockholm, and 38 in Oslo. Dispatchers still started CPR instructions in 41, 22, and 40\% of the calls, respectively and provided quality assessment in 71,100 , and $80 \%$ in these respective instances.
\end{abstract}

\footnotetext{
*Correspondence: camilla.hardeland@hiof.no; camilla.hardeland@gmail.com

'Department of Health and Welfare, Østfold University College, P.O. box 700, NO-1757 Halden, Norway

${ }^{2}$ Norwegian National Advisory Unit on Prehospital Emergency Medicine (NAKOS), Division of Prehospital Services, Oslo University Hospital and University of Oslo, Oslo, Norway

Full list of author information is available at the end of the article
}

(c) The Author(s). 2021 Open Access This article is licensed under a Creative Commons Attribution 4.0 International License, which permits use, sharing, adaptation, distribution and reproduction in any medium or format, as long as you give appropriate credit to the original author(s) and the source, provide a link to the Creative Commons licence, and indicate if changes were made. The images or other third party material in this article are included in the article's Creative Commons licence, unless indicated otherwise in a credit line to the material. If material is not included in the article's Creative Commons licence and your intended use is not permitted by statutory regulation or exceeds the permitted use, you will need to obtain permission directly from the copyright holder. To view a copy of this licence, visit http://creativecommons.org/licenses/by/4.0/ The Creative Commons Public Domain Dedication waiver (http://creativecommons.org/publicdomain/zero/1.0/) applies to the data made available in this article, unless otherwise stated in a credit line to the data. 
Conclusions: We observed variations in OHCA recognition in 71-96\% and dispatcher assisted-CPR were provided in 50-80\% in NO-CPR prior calls. In cases where CPR was initiated prior to emergency calls, dispatchers were less likely to start CPR instructions but provided quality assessments during instructions.

Keywords: Emergency medical dispatch, Cardiac arrest, Cardiopulmonary resuscitation, Cpr, Emergency medical dispatch Centre, Dispatcher, Out-of-hospital cardiac arrest

\section{Background}

Out-of-Hospital Cardiac Arrest (OHCA) affects approximately 350,000 people in Europe and 700,000 in the United States each year $[1,2]$. Incidence rates in Europe varies from 27 to 91 per 100,000 population per year [3]. The European resuscitation council (ERC) have highlighted emergency medical dispatch centres (EMDCs) as an important key player for early recognition of OHCA and in providing dispatcher assisted cardiopulmonary resuscitation (DA-CPR) before emergency medical services (EMS) arrival [1]. Early recognition of OHCA is associated with early and increased rates of bystander cardiopulmonary resuscitation (CPR) and improved survival rates [4-8]. Implementation of scripted protocols are associated with improved recognition of OHCA by emergency medical dispatchers (EMDs) $[1,5,9]$ who handle and prioritize the emergency calls. Performance goals for recognition of OHCA in EMDCs are not well established, but it is suggested that EMDCs should aim to recognise 95\% of all OHCA cases in calls where the dispatcher is able to assess consciousness and breathing. Further, recognition should be established within $1 \mathrm{~min}$ from the start of a call, and DA-CPR initiated within 2 min [10-12].

Recognition sensitivity of OHCA differ between countries and dispatch centres, with reported numbers ranging from 14 to $97 \%$, median $74 \%$ [13]. This variation may be due to the difference in EMS organisations or the definition of recognition of OHCA in the reported data. Another limitation in the comparison between existing studies may be the inclusion criteria for the OHCAs. A difference in the context of the OHCA exists and thus the premise for recognition. For example, cardiac arrests in wich bystander are already undergoing CPR at the time of the emergency call differs from OHCA where CPR is not initiated prior to the call in terms of recognition, given the bystanders awareness of the OHCA. A study has disproved the association between bystander CPR initiation before the emergency call and survival, despite the fact that the OHCA is already recognized by bystander prior to the emergency call [14]. We speculate that this lack of association illustrates a more efficient resuscitation attempt if the medical diaspatchers guide bystanders throughout the process, from recognition of the OHCA to ambulance arrival. In a study combining closed-circuit television and medical emergency calls, Linderoth et al. discovered poor quality CPR in some cases where bystanders started CPR on their own initiative [15].

Despite the high proportion of emergency calls in which bystander CPR has begun prior to the emergency call (reported as high as 35\%) [14], and despite the potential for improvement of CPR quality, little is known about callhandling in these specific emergency calls. In studies focusing on performance of DA-CPR, cases in which bystander CPR is initiated prior to the call is often excluded $[5,16,17]$ and current ERC guidelines [1] lack guidance about DA-CPR in this context.

The aim of this study is to: (1) describe OHCA call handling in EMDCs in three Scandinavian capitals with special focus on sensitivity of recognition of OHCA, provision of dispatcher-assisted CPR and time intervals and (2) to describe OHCA call handling in cases where CPR is initiated prior to the emergency call.

\section{Methods}

\section{Study design and setting}

This is an observational, multicentre study including dispatch centres in capital regions of the Scandinavian countries: Copenhagen (Denmark), Stockholm (Sweden) and Oslo (Norway). All three study sites used criteria based dispatch (CBD). In a CBD system, dispatchers rely on a decision support tool in addition to their own knowledge and experience. All sites used local adjusted versions of the third Norwegian index for emergency care [18] which instruct dispatchers to verify unconsciousness before proceeding to the protocol for "unconscious patient, not breathing normally". How to establish abnormal breathing is an individual assessment, consequently some dispatchers ask if the patient is breathing (breathing addressed), while others ask if the patient is breathing normally (abnormal breathing also addressed). The protocol further provides a structured dialogue describing CPR instructions. Repeated assessments of quality of bystander-CPR or techniques to motivate and encourage the bystander while performing CPR are not described as part of the protocol, and dispatchers make individual decisions on measures to ensure ongoing CPR of high quality. The only difference in protocol between sites, was that in Copenhagen, they ask whether or not the caller is skilled in CPR, but in Stockholm and Oslo this question was not part of the protocol. Coordinated public access Automated External Defibrillation (AED) 
programs were implemented in and linked electronically to the dispatch prioritization tool in Copenhagen and Stockholm, but not in Oslo. Study site characteristics are shown in Table 1.

\section{Copenhagen}

Copenhagen EMS has one EMDC covering the largest of five regions in Denmark. The emergency phone number 1-1-2 connects to a primary call centre that locates the address and categorises the need for police, fire department or medical assistance. In case of a medical emergency, the call is forwarded to an EMDC that answers the call, reconfirms the address and responds by activating the appropriate EMS response. The medical dispatchers are registered nurses (RN) $(70 \%)$ or paramedics $(30 \%)$ with 6 weeks of additional training in communication and the prioritization of emergency calls.

\section{Stockholm}

The EMDC in Stockholm is one of 15 nationally linked EMDCs. Dispatchers are obliged to answer 112-calls within $15 \mathrm{~s}$ in $92 \%$ of all cases. In In high call volume periods, 112-calls are automatically transferred to free dispatchers in other EMDCs throughout Sweden in order to reduce time delay in answering calls. In 2016, 20\% of the staff at the EMDC in Stockholm were RN's whilst $80 \%$ were nursing assistants or non health care providers. The training consisted of two blocks, a) 13 weeks of theory and practice followed by b) 8 weeks of supervised work. Annual re-certification is generally required for all EMDs.

\section{Oslo}

Oslo EMDC is the largest in Norway and covers both urban and rural areas. The EMDC is staffed with 46\% emergency medical technicians (EMTs)/paramedics (EMTs with 6 months further education) coordinating ambulance responses and 54\% RNs (EMDs) answering emergency calls. Training consist of 4 weeks of lectures/ theory, then approximately 2 months working under supervision of another EMD. Time spent under supervision depends on the individuals progress and prior experience.

\section{Data collection}

We identified OHCAs from the respective national cardiac arrest registries and linked the cases with data from the EMDC to obtain the audio files of the emergency calls. Inclusion criteria in the cardiac arrest registries were the same in each country; CPR initiated by either bystander or EMS. The data collection period was from January 1st, 2016 to March 17th, 2016 (Copenhagen), March 24th 2017 (Stockholm) and May 12th, 2016 (Oslo). We excluded OHCA characterized as: (1) cardiac arrests witnessed by EMS personnel ("EMS witnessed"), (2) patient alive at time of call, (3) caller cannot access patient, (4) call interrupted, and (5) audio file not available. Cases were divided in two groups: In group 1 (referred to as NO-CPR prior) CPR was NOT initiated prior to the emergency call, in group 2 (referred to as $\mathrm{CPR}_{\text {prior }}$ ), CPR was initiated by the bystander prior to the emergency call. Consecutive OHCAs were collected until $200 \mathrm{OHCA}$ cases in the NO-CPR prior group was included from each study site. Totally 200 cases from each site was deemed sufficient to provide base line characteristics in accordance with our aim. Both data collection

Table 1 Study site characteristics (2016)

\begin{tabular}{|c|c|c|c|}
\hline & $\begin{array}{l}\text { Greater Copenhagen } \\
\text { Denmark }\end{array}$ & $\begin{array}{l}\text { Stockholm region } \\
\text { Sweden }\end{array}$ & $\begin{array}{l}\text { Oslo region } \\
\text { Norway }\end{array}$ \\
\hline Population & 1.8 million & 2.3 million & 1.6 million \\
\hline Area covered by EMDC & $2568 \mathrm{~km}^{2}$ & $6519 \mathrm{~km}^{2}$ & $9551 \mathrm{~km}^{2}$ \\
\hline Incidence of OHCA & $82 / 100,000$ & $45 / 100,000$ & $61 / 100,000$ \\
\hline Incoming emergency calls 2016 & 133,772 & 206,729 & 172,934 \\
\hline $\begin{array}{l}\text { Proportion of calls resulting in an } \\
\text { ambulance response }\end{array}$ & $\begin{array}{l}\text { Priority 1: } \\
42,645 \text { (32\%) } \\
\text { Priority 2: } \\
41,528 \text { (31\%) } \\
\text { Priority } 3 \text { (non-emergency): } \\
28,281 \text { (21\%) }\end{array}$ & $\begin{array}{l}\text { Priority 1: 115,453 (56\%) } \\
\text { Priority 2: } \\
80,716 \text { (39\%) } \\
\text { Priority 3 (non-emergency): } \\
\text { 10,567 (5\%) }\end{array}$ & $\begin{array}{l}\text { Priority 1: } \\
77,460 \text { (45\%) } \\
\text { Priority 2: } \\
70,269 \text { (41\%) } \\
\text { Priority 3 (non-emergency): } \\
45,724 \text { (26\%) }\end{array}$ \\
\hline Number for emergency & 112 for medical, police, and fire/rescue & 112 for medical, police, and fire/rescue & $\begin{array}{l}113 \text { for medical, } 112 \text { for police, } \\
\text { and } 110 \text { for fire/rescue }\end{array}$ \\
\hline Medical dispatcher background & Nurse / paramedic & Nurse/Other & Nurse/paramedic \\
\hline $\begin{array}{l}\text { Specific training in handling cardiac } \\
\text { arrest calls in the dispatch centre. }\end{array}$ & Yes & Yes & Yes \\
\hline Manual/electronic use of Index & Electronic & Electronic & Manual \\
\hline
\end{tabular}


period and number of included cases in $\mathrm{CPR}_{\text {prior-group }}$ varied in the three study sites (Fig. 1).

In Stockholm, a large number of calls are handled by other EMDCs and were excluded before review $(n=$ 1425). There is a national dispatch organisation in Sweden where all calls can be handled in either of 15 different dispatch centres. This has no effect on delays or quality of DA-CPR instructions, but collection of calls from Stockholm and handled by the Stockholm dispatch centres was therefore extended in time.

A common template in combination with a detailed data dictionary was used when reviewing OHCA calls. The template and data dictionary were based on the "Cardiac Arrest Registry to Enhance Survival" (CARES) data dictionary [19], with some adjustments, see (Additional file 1) for the complete data dictionary. A total of 34 data points were collected from the audio files, focusing on recognition of cardiac arrest, time intervals, and provision of DA-CPR. Several meetings with the reviewers were held to discuss data points, inclusion criteria, terminology/definitions and difficult cases. Data collection was performed by three researchers in Copenhagen, three in Stockholm and two in Oslo. Cardiac arrest was defined as recognised if the dispatcher indicated that CPR should be performed during the call. We also registered whether the dispatcher made assessment of quality of performance of bystander-CPR during the calls, such as "please count out loud with me», «are you pushing deep enough?», «push a bit faster/slower» and if dispatchers used encouraging and motivational techniques, e.g. «keep on going», «you're doing a great job», «the ambulance is on its way».
Data points from cardiac arrest registries which were similar across all three sites were collected, such as patient characteristics, witness status, initial rhythm and ambulance treatment.

\section{Statistical analysis}

Descriptive analysis were performed using a spreadsheet program (Microsoft Excel 2019, Microsoft Corp, Redmond, WA, USA) or a statistical software package (SPSS 26.0, SPSS Inc., Chicago, IL, USA). Values are provided as numbers with percentages or medians with interquartile range. Categorical data were analysed using Pearson chi-squared test. Comparisons of continuous data were done with nonparametric Independent-Samples Kruskal-Wallis Test. PValues $\leq 0.05$ were considered significant.

\section{Results}

In order to include $200 \mathrm{OHCA}$ cases from each country (NO-CPR $\mathrm{R}_{\text {prior }}$ ), 320 calls were reviewed in Copenhagen, 235 in Stockholm, and 269 in Oslo. Cases in which CPR was initiated prior to call $\left(\mathrm{CPR}_{\text {prior }}\right)$ comprised 69 cases in Copenhagen, 9 in Stockholm, and 38 in Oslo (Fig. 1). An overview of patient and resuscitation characteristics are shown in Table 2, and dispatcher performance is shown in Table 3.

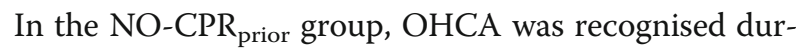
ing the call in $71 \%$ of the cases in Copenhagen, $83 \%$ in Stockholm and $96 \%$ in Oslo. CPR instructions were started in 50,60, and $80 \%$, respectively (Table 3). CPR instructions were not started in cases were cardiac arrest was not recognised, caller was unable or unwilling to perform CPR, or caller was not at scene. 'In cases where

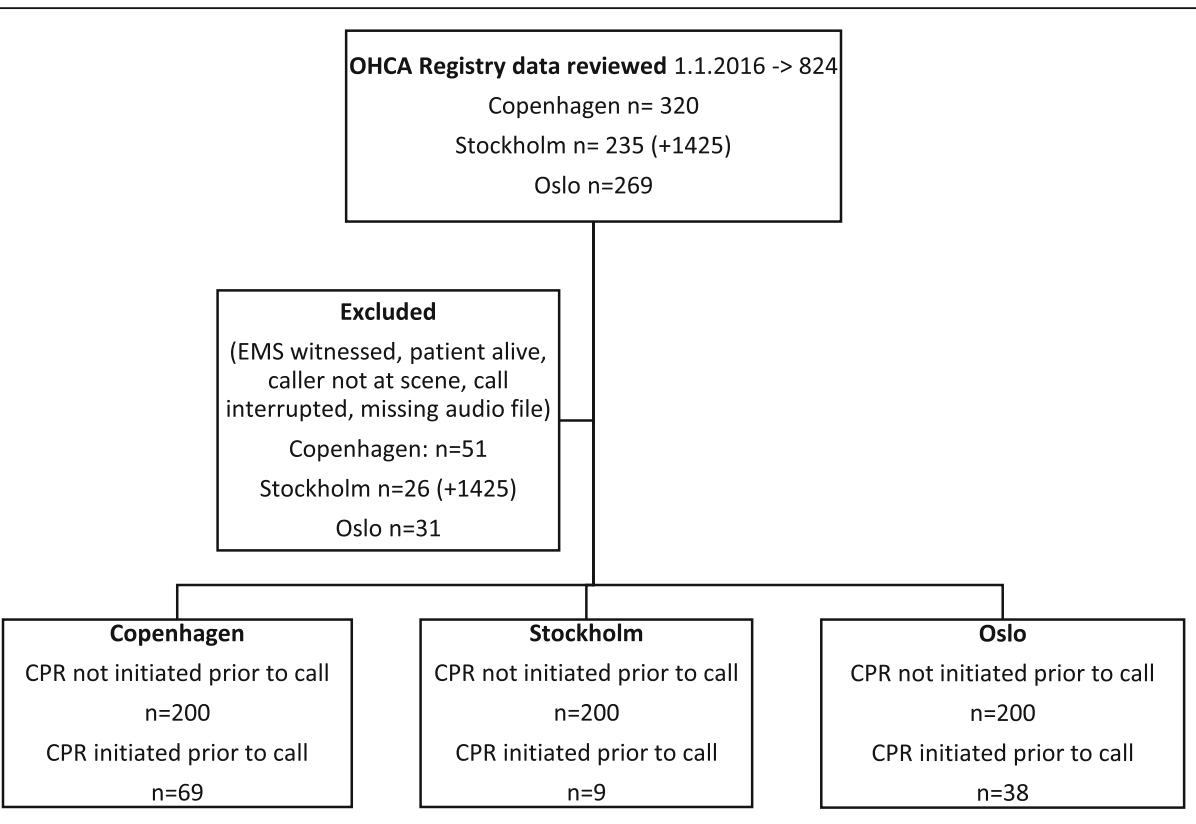

Fig. 1 Inclusion strategy 
Table 2 Patient and resuscitation characteristics of OHCA call handling

\begin{tabular}{|c|c|c|c|}
\hline Group 1: No CPR prior to call & Copenhagen $(N=200)$ & Stockholm $(N=200)$ & Oslo $(N=200)$ \\
\hline Age (years) median [IQR] & $72[62 ; 82]$ & $72[59 ; 82]$ & $65[47 ; 77]$ \\
\hline Unknown & - & $4(2)$ & - \\
\hline Male gender & $121(61)$ & $122(61)$ & $129(65)$ \\
\hline Unknown & $9(5)$ & $1(1)$ & - \\
\hline \multicolumn{4}{|l|}{ Location } \\
\hline Home & $157(79)$ & $152(76)$ & $142(71)$ \\
\hline Public & $19(10)$ & $32(16)$ & $42(21)$ \\
\hline Other & $24(12)$ & $16(8)$ & $16(8)$ \\
\hline Caller is health care personnel & $45(23)$ & $30(15)$ & $39(20)$ \\
\hline Unknown & - & $47(24)$ & $6(3)$ \\
\hline \multirow[t]{2}{*}{ Bystander witnessed } & $106(53)$ & $105(53)$ & $103(52)$ \\
\hline & - & $5(3)$ & - \\
\hline \multirow[t]{2}{*}{ Bystander CPR } & $130(65)$ & $109(55)$ & $183(92)$ \\
\hline & - & - & $4(2)$ \\
\hline Bystander defibrillation (AED) & $14(7)$ & $5(3)$ & $8(4)$ \\
\hline Initial shockable rhythm & $33(17)$ & $32(16)$ & $33(17)$ \\
\hline CPR by EMT & $179(90)$ & $189(95)$ & $138(69)$ \\
\hline Defibrillation by EMT & $47(24)$ & $64(33)$ & $45(23)$ \\
\hline Group 2: CPR initiated prior to call & Copenhagen $(N=69)$ & Stockholm $(N=9)$ & Oslo $(N=38)$ \\
\hline Age (years) median [IQ1;|Q3] & $75[60 ; 86]$ & $66[62 ; 78]$ & $66[44 ; 79]$ \\
\hline Male gender & $31(45)$ & $7(78)$ & $26(68)$ \\
\hline Unknown & $3(4)$ & - & - \\
\hline \multicolumn{4}{|l|}{ Location } \\
\hline Home & $41(59)$ & $2(22)$ & $13(34)$ \\
\hline Public & $7(10)$ & $4(44$ & $13(34)$ \\
\hline Other & $21(30)$ & $3(33)$ & $12(32)$ \\
\hline Caller is health care personnel & $39(57)$ & $4(44)$ & $17(45)$ \\
\hline Unknown & - & $4(44)$ & - \\
\hline Bystander witnessed & $30(43)$ & $4(44)$ & $24(63)$ \\
\hline Bystander defibrillation (AED) & $16(23)$ & $1(11)$ & $4(11)$ \\
\hline Initial shockable rhythm & $11(16)$ & $2(22)$ & $10(26)$ \\
\hline CPR by EMT & $56(81)$ & $8(89)$ & $26(68)$ \\
\hline Defibrillation by EMT & $14(20)$ & $5(63)$ & $11(29)$ \\
\hline Unknown & - & $1(11)$ & - \\
\hline
\end{tabular}

Values given as numbers (percentages) Abbreviations: CPR cardiopulmonary resuscitation, IQR Inter quartile range, $A E D$ automated external defibrillator, EMT Emergency medical technician

CPR instructions were given, Quality assessment was used in 80,74 , and $74 \%$ of the cases. Encouraging and/ or motivating techniques were in use in 70,83 and $83 \%$ respectively. Median time (mm:ss) to recognition of OHCA was 01:16 (Copenhagen), 01:53 (Stockholm) and 01:19 (Oslo) and median time to first chest compression was 02:35 (Copenhagen), 03:50 (Stockholm) and 02:58 (Oslo).
In the $\mathrm{CPR}_{\text {prior }}$ group, dispatchers started CPR instructions in $41 \%$ of the cases in Copenhagen, $22 \%$ in Stockholm, and $40 \%$ in Oslo. In cases where instructions were started, quality assessment was used in 71, 100, and $80 \%$ of the cases. Encouraging and/or motivating techniques were in use in 71, 100 and 93\% respectively.

Across all three sites, the $\mathrm{NO}-\mathrm{CPR}_{\text {prior }}$ and $\mathrm{CPR}_{\text {prior }}$ group comprised 600 versus 116 cases, respectively. CPR 
Table 3 Dispatcher performance

\begin{tabular}{|c|c|c|c|c|}
\hline Group 1: No CPR prior to call (CPR during) & Copenhagen $(N=200)$ & Stockholm $(N=200)$ & Oslo $(N=200)$ & $p$-value \\
\hline Consciousness addressed & $168 / 200(84)$ & $189 / 200(95)$ & 199/200 (100) & $p=0.001$ \\
\hline Unknown & 17/200 (9) & - & - & \\
\hline Breathing addressed & 169/200 (85) & $196 / 200$ (98) & 199/200 (100) & $p<0.001$ \\
\hline Unknown & 19/200 (10) & - & - & \\
\hline Abnormal breathing addressed & 68/200 (34) & $13 / 200(7)$ & 195/200 (98) & $p<0.001$ \\
\hline Unknown & $15 / 200(8)$ & $1 / 200(1)$ & - & \\
\hline OHCA recognition & $142 / 200(71)$ & 165/200 (83) & 192/200 (96) & $p<0.001$ \\
\hline Unknown & $13 / 200(7)$ & - & $1 / 200(1)$ & \\
\hline CPR instructions started & $99 / 200(50)$ & $120 / 200(60)$ & 160/200 (80) & $p<0.001$ \\
\hline Unknown & $24 / 200(12)$ & - & - & \\
\hline Dispatcher is assertive when providing CPR instructions & $84 / 99(85)$ & 101/120 (84) & $145 / 160(91)$ & $p=0.2$ \\
\hline Unknown & - & $3 / 120(3)$ & - & \\
\hline Quality assessment & $79 / 99(80)$ & $89 / 120(74)$ & $119 / 160(74)$ & $p=0.2$ \\
\hline Unknown & - & $7 / 120(6)$ & - & \\
\hline Encouraging/motivating techniques in use & $69 / 99(70)$ & $99 / 120(83)$ & $132 / 160(83)$ & $p<0.001$ \\
\hline Chest compressions performed & 109/200 (55) & $119 / 200(60)$ & 157/200 (79) & $p<0.001$ \\
\hline Unknown & - & $5 / 200(3)$ & $4 / 200(2)$ & \\
\hline Type of CPR & & & & $p=0.3$ \\
\hline $30: 2$ & 24/109 (22) & $30 / 119(25)$ & 46/157 (29) & \\
\hline Compressions only & $85 / 109(78)$ & $77 / 119(65)$ & 102/157 (65) & \\
\hline Unknown & - & 20/119 (17) & 9/157 (6) & \\
\hline BLS competence addressed & $88 / 200(44)$ & $101 / 200(51)$ & $63 / 200(32)$ & $p<0.001$ \\
\hline Unknown & $30 / 200(15)$ & $1 / 200(1)$ & - & \\
\hline AED addressed & $22 / 200(11)$ & $8 / 200(4)$ & $6 / 200(3)$ & $p<0.001$ \\
\hline Unknown & $51 / 200(26)$ & - & - & \\
\hline Call continued until EMS arrival & $85 / 200(43)$ & $128 / 200(64)$ & $163 / 200(82)$ & $p<0.001$ \\
\hline Unknown & $14 / 200(7)$ & - & $3 / 200(2)$ & \\
\hline \multicolumn{5}{|l|}{ Time intervals for recognition and CPR instructions } \\
\hline Time to OHCA recognition (min:sec) & 01:16 [IQR 00:50-02:11] & 01:53 [IQR 1:01-3:13] & 01:19 [IQR 0:50-2:09] & $p<0.001$ \\
\hline Time to chest compression instructions (min) & 02:10 [IQR 01:27-03:25] & 03:20 [IQR 02:03-04:56] & 02:24 [IQR 01:37-04:00] & $p<0.001$ \\
\hline Time to chest compressions performed (min) & 02:35 [IQR 01:45-03:05] & 03:50 [IQR 02:30-05:27] & 02:58 [IQR 02:09-04:36] & $p<0.001$ \\
\hline Group 2: CPR initiated prior to call $\left(\mathrm{CPR}_{\text {prior }}\right)$ & Copenhagen $(N=69)$ & Stockholm $(N=9)$ & Oslo $(N=38)$ & \\
\hline CPR instructions started & $28 / 69(41)$ & 2/9 (22) & $15 / 38(40)$ & \\
\hline Unknown & $8 / 69(12)$ & - & - & \\
\hline Dispatcher is assertive when providing CPR instructions & $24 / 28(86)$ & $2 / 2(100)$ & 15/15 (100) & \\
\hline Quality assessment & 20/28 (71) & $2 / 2(100)$ & $12 / 15(80)$ & \\
\hline Encouraging/motivating techniques in use & $20 / 28(71)$ & $2 / 2(100)$ & 14/15 (93) & \\
\hline \multicolumn{5}{|l|}{ Type of CPR } \\
\hline $30: 2$ & $17 / 69(25)$ & $1 / 9(11)$ & $7 / 38(18)$ & \\
\hline Compressions only & $30 / 69(43)$ & $1 / 9(11)$ & $12 / 38(32)$ & \\
\hline Unknown & $22 / 69(32)$ & $7 / 9(78)$ & $19 / 38(50)$ & \\
\hline AED addressed & 27/69 (39) & 3/9 (33) & $4 / 38(11)$ & \\
\hline Call continued until EMS arrival & $25 / 69(36)$ & 4/9 (44) & $19 / 38(50)$ & \\
\hline
\end{tabular}


instructions were started in $63 \%$ in $\mathrm{NO}-\mathrm{CPR}_{\text {prior }}$ and $39 \%$ in $\mathrm{CPR}_{\text {prior. In }}$ cases were $\mathrm{CPR}$ instructions were started, quality assessment and encouraging and/or motivational techniques were similarly used in the two groups. Quality assessment was found in 76\% of cases in both groups, and encouraging and/or motivational techniques were found in $79 \%$ of the cases in NO-CPRprior and $80 \%$ in the CPRprior group. The caller was a health care personnel in $19 \%$ in $\mathrm{NO}-\mathrm{CPR}_{\text {prior }}$ and $52 \%$ in $\mathrm{CPR}_{\text {prior }}$ group.

\section{Discussion}

The main results of this study show that OHCA recognition rates in cases were no CPR was initiated prior to call varied from 71 to $96 \%$ between three capital city EMDCs in Scandinavia. Time to recognition varied by over half a minute, and time to first chest compression varied by over a minute. We question whether this is due to differences in dispatcher performance or differences in systems as system variables were difficult to compare objectively.

\section{OHCA recognition}

International literature shows extensive variations in recognition sensitivity of OHCA. 13]. Reasons for the significant difference in OHCA recognition in this study is multifaceted. Oslo had $1 \frac{1}{2}$ years prior to this study undergone a targeted intervention to improve performance in recognition rates and DA-CPR [20]. 'Attention to appropriate handling of cardiac arrest calls was an ongoing priority in the EMDC in 2016, which may have affected the high level of recognition in Oslo. The ERC guidelines 2015 state that dispatchers can improve recognition by focusing on "unresponsiveness" and "not breathing normally" [1]. All three sites addressed consciousness and breathing in most cases, but Oslo was the only site also addressing abnormal breathing in most cases. This might be the main reason for differences in recognition rates between the three countries.

Differences in educational levels or professional background of the dispatchers might also be factors impacting on recognition rates. In Stockholm, EMDs were not necessarily health care personnel, and only $20 \%$ were nurses. In Oslo and Copenhagen, EMDs were nurses or paramedics.

Dispatcher performance can directly affect OHCA recognition rates by including false OHCA cases in the cardiac arrest registry. Inclusion criteria in the cardiac arrest registries in the Scandinavian countries are the same; all cases where anyone at scene (bystander or EMTs) have started CPR, is included in the registry. But if callers are instructed to start CPR in unclear cases where the patient is actually not in cardiac arrest, they are still included in the registry. Hence, pro-active dispatchers can increase the incidence numbers in the cardiac arrest registries by providing unnecessary CPR instructions. Although not reported in this study, these cases might also affect outcome data, possibly including both cases were the patient was never in cardiac arrest, as well as cases where no treatment was started by EMT due to futility.

Despite a high proportion of recognised cases in all three sites, and AEDs mentioned in the protocol, AEDs were rarely addressed in the NO-CPR prior group. Reasons for this is unknown, but a study from Sweden exploring this specifically found that AEDs were not nearby in $93 \%$ of the cases. Other reasons might be inaccessible AEDs and caller being alone [21]. There were significant differences between sites on AED addressed in this study (11\% in Copenhagen, $4 \%$ in Stockholm and $3 \%$ in Oslo). Longstanding efforts from the Danish AED registry might have affected this. Oslo did not have a functional AED registry at the time of data collection.

There was seemingly a relatively long time interval from OHCA recognition to start of first chest compression instruction in all sites (00,54 in Copenhagen, 01:27 in Stockholm, 01:05 in Oslo). This was due to the Cares definition of time interval for "chest compression instruction", which states that "Instructions to get a patient to a hard, flat surface should not be considered the start of CPR instructions. Instructions begin when a calltaker or dispatcher tells the rescuer to "kneel by the patient's side."” [19].

\section{CPR initiated prior to the emergency call}

There were great variations in proportions of cases were CPR was initiated prior to call in the three study sites (69 (Copenhagen) versus 9 (Stockholm) versus 38 (Oslo)). Reasons for this might be differences in basic life support (BLS) programs or organisational differences, in Copenhagen all calls are handled by the police before transferred to a medical dispatcher, providing more time for qualified bystanders to initiate CPR. Results from this study indicate that dispatchers are less likely to provide CPR instructions to callers when CPR is initiated prior to the emergency call. Early CPR is associated with increased survival [22-24] but favourable outcome depends on high quality CPR performance [25]. Studies comparing DA-assisted CPR and bystander initiated CPR prior to the call have found no significant difference in survival between the two groups [13, 26]. However, Takei et al. showed significantly more good quality CPR compared to low quality CPR (OR 2.67) in bystander-initiated CPR prior to the call to the EMDC [27]. It is fair to assume that bystanders who start CPR without instructions are at least willing to perform CPR. Their skills and abilities are uncertain at the time of the emergency call and might be clarified by the medical 
dispatchers who in addition can support the bystander in performing high quality CPR. Lack of CPR instructions to bystanders performing CPR prior to call indicates a knowledge gap. There is a need for further exploration of the consequences when dispatchers do not provide CPR instructions to a large group of bystanders in a group mostly excluded from studies on DA-CPR.

A high proportion of health care personnel as callers might explain why dispatchers to a lesser extent provide $\mathrm{CPR}$ instructions in cases where CPR is initiated prior to the call. Health care professional bystanders are more likely to initiate CPR prior to the call [13]. CPR performed by health care professionals has been shown to have increased patient survival compared to bystander initiated CPR performed by laypeople [28, 29], but a recent study showed no such difference [30]. It can be difficult for dispatchers to assess callers' competence in CPR based on the fact that they are health care providers. For example, a common situation in OHCA is that the patient is discovered by representatives from the home care services, and the caller is perceived by the dispatcher to be a health care provider. The home care services can be staffed by people with no or limited medical training [31] and potentially no experience in handling a cardiac arrest patient, hence in as much need of CPR instructions as lay people.

When CPR is initiated prior to the call to the EMDC, cases are recommended to be excluded from review in studies reporting OHCA recognition rates, [13, 17]. These cases are rarely described, even though this seems necessary in order to give proper recommendations for dispatchers on handling these cases. In the future it is reasonable to include more information on the interaction in the first resuscitation team in BLS-programs. Likewise, terminology should be standardised between national CPR councils providing course curriculums and dispatch organisations decision and prioritisation tools, also in cases where CPR is initiated prior to emergency calls. To avoid unnecessary complications and ensure optimal treatment of all OHCA patients, we suggest that dispatchers always provide CPR instructions (also to health care personnel and when CPR is initiated prior to call), make quality assessments during the call and stay on the line until EMS arrival.

\section{Limitations}

This study has several limitations. There are few cases where CPR is initiated prior to the call, and the numbers vary between the three study sites. More data would have made comparisons possible. These data should therefore be regarded as explorative findings providing hypothesis generating knowledge on a group rarely described in other studies. A low number of cases from each site and potential differences in BLS-programs may interfere with the analysis. Inclusion and outcome data is affected by dispatcher performance between sites, and differences in system organisations make comparisons between sites difficult. For example OHCA incidence (per 100,000 inhabitants) in the registries differ from 45 (Stockholm), 61 (Oslo) and 85 (Copenhagen), and CPR by EMT is lower in Oslo than Stockholm and Copenhagen. There is no reason to believe that there are great variations in morbidity between countries, and explanations to these variations might be found in inclusion criteria and reporting rate to the cardiac arrest registries. Time differences between sites are also likely to differ due to differences in call handling. In Denmark the emergency call is initially answered by the police who then refers the call to the EMDC, time variable is measured from EMDC taking the call. In Stockholm the EMDC answer all emergency calls, and $70 \%$ of calls are not medical emergencies. In Oslo there is a specific telephone number for medical emergencies only.

High recognition rate may be associated with a higher false positive rate. This is important because of scarce resources which influences management decisions. We have not been able to establish a false positive rate in this study. When reviewing audio files there will always be some interpretation by the reviewer. We tried to minimize this issue by a detailed data extraction protocol. Despite meticulous preparations, there are cases not possible to determine accurately from audio files, resulting in more unknown cases than expected (Table 3). Reasons for this were most often if callers were excessively distraught, (in quite a few cases the caller left the phone) or there were language barriers. Reviewers from respective countries did not have access to other countries' audio files, and no interrater agreement across countries could be performed.

\section{Conclusions}

In this study, we used a common template to collect data from EMDCs with seemingly similar health systems in the three Scandinavian capital regions. However, the main findings show variations in OHCA recognition and provision of DA-CPR, and we question whether this is due to differences in dispatcher performance or differences in systems. Further exploration of reasons for these variations are necessary. Descriptions of calls where CPR was initiated prior to contacting the EMDCs indicate that callers are more likely to be health care personnel, and dispatchers are less likely to provide CPR instructions to such callers. Further studies to explore the consequences of not providing CPR instructions to bystanders are needed. 


\section{Abbreviations}

AED: Automated External Defibrillator; CARES: Cardiac Arrest Registry to Enhance Survival; OHCA: Out-of-hospital cardiac arrest; ERC: The European resuscitation council; EMDC: Emergency medical dispatch centre; DACPR: Dispatcher assisted cardiopulmonary resuscitation; EMS: Emergency medical services; EMD: Emergency medical dispatcher; RN: Registered nurse

\section{Supplementary Information}

The online version contains supplementary material available at https://doi. org/10.1186/s13049-021-00903-4.

\section{Additional file 1.}

\section{Acknowledgements}

We would like to thank all dispatchers and EMS leaders in the three EMDCs where the study was performed, the COSTA group for inspiration and support in planning and performance of this study and Laerdal Foundation for Acute Medicine for funding parts of the study. We would also like to thank the CARES program for providing us with the template we based our data collection on

\section{Authors' contributions}

$\mathrm{CH}, \mathrm{AC}, \mathrm{MTB}, \mathrm{SNFB}, \mathrm{FF}, \mathrm{JH}, \mathrm{JK}-\mathrm{J}, \mathrm{FL}, \mathrm{AN}, \mathrm{AMN}, \mathrm{TMO}, \mathrm{MR}$, LS and TPM have made substantial contributions to conception and design of the study. $\mathrm{CH}, \mathrm{AC}, \mathrm{AMN}$, AN, SNFB and TPM have made substantial contributions in acquisition of data. $\mathrm{CH}, \mathrm{AC}, \mathrm{SNFB}$ and TPM have made substantial contributions in analysis and interpretation of data. $\mathrm{CH}, \mathrm{AC}$ and TPM have drafted the article. $\mathrm{CH}, \mathrm{AC}, \mathrm{MTB}$, SNFB, FF, JH, JK-J, FL, AN, AMN, TMO, MR, LS and TPM have substantively revised the article. CH, AC, MTB, SNFB, FF, JH, JK-J, FL, AN, AMN, TMO, MR, LS and TPM read and approved the final manuscript.

\section{Funding}

$\mathrm{CH}$ has received an unrestricted research grant from Laerdal Foundation funding parts of the study.

\section{Availability of data and materials}

Not applicable.

\section{Declarations}

\section{Ethics approval and consent to participate}

The study was conducted in accordance with principles from the Declaration of Helsinki [32] and appropriate approvals from each country were collected. In Norway exception from confidentiality was approved by the regional research ethics committee (Reference no. 2017/1621). In addition, the study was approved by the local data protection authority at Oslo University Hospital (reference no. 17/20651). In Sweden from the regional ethical board in Stockholm (reference number: 2017/2356-31/1). In Denmark the study was approved by the Danish Health Authority (3-3013-1289/1/), and the Danish Data Protection Agency (2012-58-0004) According to approvals from all three sites, data was obtained without informed consent.

\section{Consent for publication}

Not applicable.

\section{Competing interests}

AC, MTB, SNFB, FF, JH, JK-J, FL, AN, AMN, MR, LS and TPM have no competing interests. TMO has received unrestricted research funding from Zoll Foundation and Laerdal Foundation.

\section{Author details}

${ }^{1}$ Department of Health and Welfare, Østfold University College, P.O. box 700, NO-1757 Halden, Norway. ${ }^{2}$ Norwegian National Advisory Unit on Prehospital Emergency Medicine (NAKOS), Division of Prehospital Services, Oslo University Hospital and University of Oslo, Oslo, Norway. ${ }^{3}$ Department of Clinical Science and Education, Södersjukhuset, Centre for Resuscitation Science, Karolinska Institutet, Stockholm, Sweden. ${ }^{4}$ Department of Cardiology, Heart Centre, Amsterdam UMC, Academic Medical Centre, University of Amsterdam, Amsterdam, The Netherlands. ${ }^{5}$ Copenhagen Emergency Medical
Services, University of Copenhagen, Copenhagen, Denmark. ${ }^{6}$ Department of Anesthesiology, Oslo University Hospital, Oslo, Norway. ${ }^{7}$ Department of Medicine, Centre for Resuscitation Science, Karolinska Institutet, Stockholm, Sweden.

Received: 29 November 2020 Accepted: 11 June 2021

Published online: 30 June 2021

\section{References}

1. Perkins G, Handley A, Koster RW, Castren M, Smyth MA, Olasveengen T, et al. European resuscitation council guidelines for resuscitation 2015 section 2. Adult basic life support and automated external defibrillation. Resuscitation. 2015;95:81-99. https://doi.org/10.1016/j.resuscitation.2015.07. 015.

2. Mozaffarian JD, Benjamin SE, Go KA, Arnett JD, Blaha JM, Cushman JM, et al. Heart disease and stroke statistics - 2015 update: a report from the American Heart Association. Circulation. 2015;131:e29-e322.

3. Gräsner J-T, Whent J, Herlitz J, Perkins GD, Lefering R, Tjelmeland I, et al. Survival after out-of-hospital cardiac arrest in Europe - results of the EuReCa TWO study. Resuscitation. 2020;148:218-26. https://doi.org/10.1016/j. resuscitation.2019.12.042

4. Berdowski J, Beekhuis F, Zwinderman AH, Tijssen JGP, Koster RW. Importance of the first link: description and recognition of an out-ofhospital cardiac arrest in an emergency call. Circulation. 2009;119(15):2096102. https://doi.org/10.1161/CIRCULATIONAHA.108.768325.

5. Bobrow BJ, Spaite DW, Vadeboncoeur TF, Hu C, Mullins T, Tormala W, et al. Implementation of a regional telephone cardiopulmonary resuscitation program and outcomes after out-of-hospital cardiac arrest. JAMA Cardiol. 2016;1(3):294-302. https://doi.org/10.1001/jamacardio.2016.0251.

6. Vaillancourt C, Verma A, Trickett J, Crete D, Beaudoin T, Nesbitt L, et al. Evaluating the effectiveness of dispatch-assisted cardiopulmonary resuscitation instructions. Acad Emerg Med. 2007;14(10):877-83. https://doi. org/10.1197/j.aem.2007.06.021.

7. Ko SY, Shin SD, Ro YS, Song KJ, Hong KJ, Park JH, et al. Effect of detection time interval for out-of-hospital cardiac arrest on outcomes in dispatcherassisted cardiopulmonary resuscitation: a nationwide observational study. Resuscitation. 2018;129:61-9. https://doi.org/10.1016/j.resuscitation.2018.06. 002.

8. Nikolaou N, Dainty KN, Couper K, Morley P, Tijssen J, Vaillancourt C, et al. A systematic review and meta-analysis of the effect of dispatcher-assisted CPR on outcomes from sudden cardiac arrest in adults and children. Resuscitation. 2019;138:82-105. https://doi.org/10.1016/j.resuscitation.2019. 02.035.

9. Dami F, Fuchs V, Praz L, Vader JP. Introducing systematic dispatcher-assisted cardiopulmonary resuscitation (telephone-CPR) in a non-advanced medical priority dispatch system (AMPDS): implementation process and costs. Resuscitation. 2010;81(7):848-52. https://doi.org/10.1016/j.resuscitation.2010. 03.025 .

10. Lewis AM, Stubbs SB, Eisenberg SM. Dispatcher-assisted cardiopulmonary resuscitation: time to identify cardiac arrest and deliver chest compression instructions. Circulation. 2013;128(14):1522-30. https://doi.org/10.1161/ CIRCULATIONAHA.113.002627.

11. Lerner EB, Rea TD, Bobrow BJ, Acker JE, Berg RA, Brooks SC, et al. Emergency medical service dispatch cardiopulmonary resuscitation prearrival instructions to improve survival from out-of-hospital cardiac arrest: a scientific statement from the American heart association. Circulation. 2012; 125(4):648-55. https://doi.org/10.1161/CIR.0b013e31823ee5fc.

12. Bobrow BJ, Panczyk M, Subido C. Dispatch-assisted cardiopulmonary resuscitation: the anchor link in the chain of survival. Curr Opin Crit Care. 2012;18(3):228-33. https://doi.org/10.1097/MCC.0b013e328351736b.

13. Viereck S, Møller TP, Rothman JP, Folke F, Lippert FK. Recognition of out-ofhospital cardiac arrest during emergency calls - a systematic review of observational studies. Scandinavian journal of trauma, resuscitation and. Emerg Med. 2017;25:9.

14. Viereck S, Palsgaard Møller T, Kjær Ersbø\|l A, Folke F, Lippert F. Effect of bystander CPR initiation prior to the emergency call on ROSC and 30 day survival —an evaluation of 548 emergency calls. Resuscitation. 2017;111:5561. https://doi.org/10.1016/j.resuscitation.2016.11.020.

15. Linderoth G, Hallas P, Lippert FK, Wibrandt I, Loumann S, Møller TP, et al. Challenges in out-of-hospital cardiac arrest - a study combining closed- 
circuit television (CCTV) and medical emergency calls. Resuscitation. 2015;96: 317-22. https://doi.org/10.1016/.j.resuscitation.2015.06.003.

16. Hardeland C, Sunde K, Ramsdal H, Hebbert SR, Soilammi L, Westmark F, et al. Factors impacting upon timely and adequate allocation of prehospital medical assistance and resources to cardiac arrest patients. Resuscitation. 2016;109:56-63. https://doi.org/10.1016/.resuscitation.2016.09.027.

17. Dameff C, Vadeboncoeur T, Tully J, Panczyk M, Dunham A, Murphy R. A standardized template for measuring and reporting telephone pre-arrival cardiopulmonary resuscitation instructions. Resuscitation. 2014;85(7):869-73. https://doi.org/10.1016/j.resuscitation.2014.02.023.

18. The Norwegian Medical Association. Norwegian index for emergency care. Stavanger: Laerdal Medical; 2009.

19. CARES. Dispatcher-Assisted CPR Data Dictionary. 2015. https://mycares.net/ sitepages/dispatchtraining.jsp. Accessed 15 Jan 2018.

20. Hardeland C, Skåre C, Kramer-Johansen J, Birkenes TS, Myklebust H, Hansen $\mathrm{AE}$, et al. Targeted simulation and education to improve cardiac arrest recognition and telephone assisted CPR in an emergency medical dispatch Centre. Resuscitation. 2017;114:21-6. https://doi.org/10.1016/j.resuscitation.2 017.02.013.

21. Fredman D, Svensson L, Ban Y, Jonsson M, Hollenberg J, Nordberg P, et al. Expanding the first link in the chain of survival - experiences from dispatcher referral of callers to AED locations. Resuscitation. 2016:107:12934. https://doi.org/10.1016/j.resuscitation.2016.06.022.

22. Hasselqvist-Ax I, Riva G, Herlitz J, Rosenqvist M, Hollenberg J, Nordberg P. et al. Early cardiopulmonary resuscitation in out-of-hospital cardiac arrest. N Engl J Med. 2015;372(24):2307-15. https://doi.org/10.1056/NEJMoa1405796.

23. Wissenberg M, Lippert FK, Folke F, Weeke P, Hansen CM, Christensen EF. Association of national initiatives to improve cardiac arrest management with rates of bystander intervention and patient survival after out-ofhospital cardiac arrest. JAMA. 2013;310:1377-84. https://doi.org/10.1001/ja ma.2013.278483.

24. Sasson C, Rogers MA, Dahl J, Kellermann AL. Predictors of survival from outof-hospital cardiac arrest: a systematic review and meta-analysis. Circ Cardiovasc Qual Outcomes. 2010;3(1):63-81. https://doi.org/10.1161/ CIRCOUTCOMES.109.889576.

25. Talikowska M, Tohira H, Finn J. Cardiopulmonary resuscitation quality and patient survival outcome in cardiac arrest: a systematic review and metaanalysis. Resuscitation. 2015;96:66-77. https://doi.org/10.1016/j.resuscitation.2 015.07.036.

26. Takahashi H, Sagisaka R, Natsume Y, Tanaka S, Takyu H, Tanaka H. Does dispatcher-assisted CPR generate the same outcomes as spontaneously delivered bystander CPR in Japan? Am J Emerg Med. 2018;36(3):384-91. https://doi.org/10.1016/j.ajem.2017.08.034.

27. Takei Y, Nishi T, Matsubara H, Hashimoto M, Inaba H. Factors associated with quality of bystander CPR: the presence of multiple rescuers and bystander-initiated CPR without instruction. Resuscitation. 2014;85(4):492-8. https://doi.org/10.1016/j.resuscitation.2013.12.019.

28. Herlitz J, Svensson L, Holmberg S, Ängquist K-A, Young M. Efficacy of bystander CPR: intervention by lay people and by health care professionals. Resuscitation. 2005;66(3):291-5. https://doi.org/10.1016/.resuscitation.2005. 04.003.

29. Nord A, Svensson L, Karlsson T, Claesson A, Herlitz J, Nilsson L. Increased survival from out-of-hospital cardiac arrest when off duty medically educated personnel perform CPR compared with laymen. Resuscitation. 2017;120:88-94. https://doi.org/10.1016/j.resuscitation.2017.08.234.

30. Kobayashi D, Kitamura T, Kiyohara K, Nishiyama C, Hayashida S, Matsuyama T, et al. Cardiopulmonary resuscitation performed by off-duty medical professionals versus laypersons and survival from out-of-hospital cardiac arrest among adult patients. Resuscitation. 2019;135:66-72. https://doi.org/1 0.1016/j.resuscitation.2019.01.005.

31. Gautun H. BC. Rapport nr. 14/14: Bemanning og kompetanse i hjemmesykepleien og sykehjem. Oslo: Velferdsforskningsinstituttet NOVA; 2014.

32. World Medical Association. World Medical Association Declaration of Helsinki: ethical principles for medical research involving human subjects. JAMA. 2013;310(20):2191-4. https://doi.org/10.1001/jama.2013.281053.

\section{Publisher's Note}

Springer Nature remains neutral with regard to jurisdictional claims in published maps and institutional affiliations.

Ready to submit your research? Choose BMC and benefit from:

- fast, convenient online submission

- thorough peer review by experienced researchers in your field

- rapid publication on acceptance

- support for research data, including large and complex data types

- gold Open Access which fosters wider collaboration and increased citations

- maximum visibility for your research: over $100 \mathrm{M}$ website views per year

At BMC, research is always in progress.

Learn more biomedcentral.com/submissions 